Acta Crystallographica Section E

Structure Reports

Online

ISSN 1600-5368

Xianghong Huang, ${ }^{a}$ Qian-Feng Zhang $^{\text {b* }}$ and Herman H. Y. Sung ${ }^{c}$

${ }^{a}$ College of Applied Technology, Wenzhou University, Wenzhou, Zhejiang 325035 , People's Republic of China,

${ }^{\mathbf{b}}$ Department of Chemistry and Chemical Engineering, Anhui University of Technology, Maanshan, Anhui 243002, People's Republic of China, and ' Department of Chemistry, Hong Kong University of Science and Technology, Clear Water Bay, Kowloon, Hong Kong,

People's Republic of China

Correspondence e-mail: zhangqf@ahut.edu.cn

\section{2-(2,2,2-Trifluoroacetylamino)pyridin-3-yl trifluoromethanesulfonate. Addendum}

Owing to unfortunate circumstances the paper by Huang, Zhang \& Sung [Acta Cryst. (2004), E60, o708-0710] reports the same structure as the paper by Huang, Liu, Hu \& $\mathrm{Ng}$ [Acta Cryst. (2004), E60, 0308-0309] and hence the two papers should be read together. The authors of the later paper apologise unreservedly for this problem. 